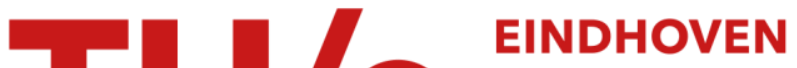 UNIVERSITY OF TECHNOLOGY
}

\section{Laboratory experiments on multipolar vortices in a rotating fluid}

Citation for published version (APA):

Trieling, R. R., Heijst, van, G. J. F., \& Kizner, Z. (2010). Laboratory experiments on multipolar vortices in a rotating fluid. Physics of Fluids, 22(9), 094104-1/12. [094104]. https://doi.org/10.1063/1.3481797

DOI:

10.1063/1.3481797

Document status and date:

Published: 01/01/2010

Document Version:

Publisher's PDF, also known as Version of Record (includes final page, issue and volume numbers)

Please check the document version of this publication:

- A submitted manuscript is the version of the article upon submission and before peer-review. There can be important differences between the submitted version and the official published version of record. People interested in the research are advised to contact the author for the final version of the publication, or visit the $\mathrm{DOI}$ to the publisher's website.

- The final author version and the galley proof are versions of the publication after peer review.

- The final published version features the final layout of the paper including the volume, issue and page numbers.

Link to publication

\section{General rights}

Copyright and moral rights for the publications made accessible in the public portal are retained by the authors and/or other copyright owners and it is a condition of accessing publications that users recognise and abide by the legal requirements associated with these rights.

- Users may download and print one copy of any publication from the public portal for the purpose of private study or research.

- You may not further distribute the material or use it for any profit-making activity or commercial gain

- You may freely distribute the URL identifying the publication in the public portal.

If the publication is distributed under the terms of Article $25 \mathrm{fa}$ of the Dutch Copyright Act, indicated by the "Taverne" license above, please follow below link for the End User Agreement:

www.tue.nl/taverne

Take down policy

If you believe that this document breaches copyright please contact us at:

openaccess@tue.nl

providing details and we will investigate your claim. 


\title{
Laboratory experiments on multipolar vortices in a rotating fluid
}

\author{
R. R. Trieling, ${ }^{1, a)}$ G. J. F. van Heijst, ${ }^{1}$ and Z. Kizner ${ }^{2}$ \\ ${ }^{1}$ Fluid Dynamics Laboratory, Eindhoven University of Technology, \\ P.O. Box 513, Eindhoven 5600 MB, The Netherlands \\ ${ }^{2}$ Departments of Mathematics and Physics, Bar-Ilan University, Ramat-Gan 52900, Israel
}

(Received 11 April 2010; accepted 3 August 2010; published online 16 September 2010)

\begin{abstract}
The instability properties of isolated monopolar vortices have been investigated experimentally and the corresponding multipolar quasisteady states have been compared with semianalytical vorticity-distributed solutions to the Euler equations in two dimensions. A novel experimental technique was introduced to generate unstable monopolar vortices whose nonlinear evolution resulted in the formation of multipolar vortices. Dye-visualization and particle imaging techniques revealed the existence of tripolar, quadrupolar, and pentapolar vortices. Also evidence was found of the onset of hexapolar and heptapolar vortices. The observed multipolar vortices were found to be unstable and generally broke up into multipolar vortices of lesser complexity. The characteristic flow properties of the quadrupolar vortex were in close agreement with the semianalytical model solutions. Higher-order multipolar vortices were observed to be susceptible to strong inertial oscillations. (C) 2010 American Institute of Physics. [doi:10.1063/1.3481797]
\end{abstract}

\section{INTRODUCTION}

In the last few decades, a large number of studies has been devoted to the origin and stability of two-dimensional multipolar vortex structures with zero total circulation. In the mid-1980s, Gent and McWilliams ${ }^{1}$ provided numerical evidence of the existence of tripolar vortices. The tripolar vortex is characterized by a collinear arrangement of three regions of alternating vorticity: a core of single-signed vorticity and two satellites of opposite vorticity where the whole ensemble rotates steadily about the center of the vortex core. Normalmode analyses ${ }^{2-4}$ confirmed that the tripolar vortex may result from the growth of a wavenumber- 2 perturbation of an unstable isolated circular monopolar vortex. In this paper the term "isolated" refers to the zero net circulation of the vortex. The spontaneous formation of a tripolar vortex has been observed and analyzed in many other numerical studies. ${ }^{5-11}$ The first experimental evidence of a tripolar vortex was given by van Heijst and Kloosterziel. ${ }^{12}$ This study was soon followed by other observations of tripolar vortices in the laboratory ${ }^{13-16}$ and in satellite imagery. ${ }^{17}$ Higher-order vortices are less commonly observed. Numerical simulations ${ }^{9,18}$ and laboratory experiments ${ }^{14,19-22}$ revealed that quadrupolar vortex structures may form due to the growth of a wavenumber-3 instability. Apart from a few numerical cases 9 we are not aware of any laboratory examples of higher-order vortex structures that arise spontaneously from an initially isolated circular vortex. This fact is consistent with theoretical studies in which it is argued that isolated multipolar vortex structures with more than three satellites are essentially unstable. ${ }^{9,19,23-26}$

The existence of multipolar vortex structures has motivated researchers to derive multipolar steady-state solutions to the Euler equations. Steady-state solutions for multipolar patches of uniform vorticity were obtained by Polvani and

${ }^{a)}$ Electronic mail: r.r.trieling@tue.nl. URL: www.fluid.tue.nl.
Carton $^{6}$ and Morel and Carton. ${ }^{9}$ Explicit regular solutions for tripolar and quadrupolar vortices with distributed vorticity and zero total circulation were first obtained by Kizner and Khvoles. ${ }^{27,28}$ In their work, the geometrical structure and the solution outside the region of nonzero vorticity were derived analytically, whereas the problem for the interior region was solved numerically. It was shown that these semianalytical solutions reproduce the main features of experimental tripoles ${ }^{13,14}$ and numerical quadrupoles ${ }^{29}$ concerning their shape, flow pattern, and the form of the vorticitystreamfunction relationship. This work was later extended by Kizner et $a l .{ }^{30}$ in order to construct semianalytical solutions for multipolar vortices with more satellite vortices and to incorporate the quasigeostrophic equivalent-barotropic ( $1 \frac{1}{2}$-layer) and $\gamma$-plane approximations.

The present study aims at finding such multipolar structures with zero total circulation in the laboratory. The experiments are carried out in a rotating tank in which the flow is quasi-two-dimensional. In order to generate multipolar vortices, we introduce a novel experimental technique which is inspired by the normal-mode analysis of Flierl. ${ }^{2}$ Within a contour-dynamics ${ }^{31}$ framework, Flierl derived analytical criteria for instability of barotropic and baroclinic circular monopoles with piecewise-uniform vorticity. A vortex of this kind consists of a core of uniform vorticity, an annulus of uniform but different vorticity, and an outer region of zero vorticity. The circular boundaries demarcating the different regions of vorticity were taken to be at the nondimensional radii $r=1$ and $r=b$. Depending on the relative width of the annulus, the vortex was found to be stable or unstable to perturbations proportional to $\exp \left[\operatorname{im}\left(\theta-\Omega_{p} t\right)\right]$, with $m$ as the azimuthal mode number and $\Omega_{p}$ as the frequency of the perturbation. For barotropic monopoles with zero net circulation (which are the focus of our study) subjected to perturbations of their boundary contours, it was found that the monopole is unstable at $b<2$, and the smaller the radius $b$, the higher the 


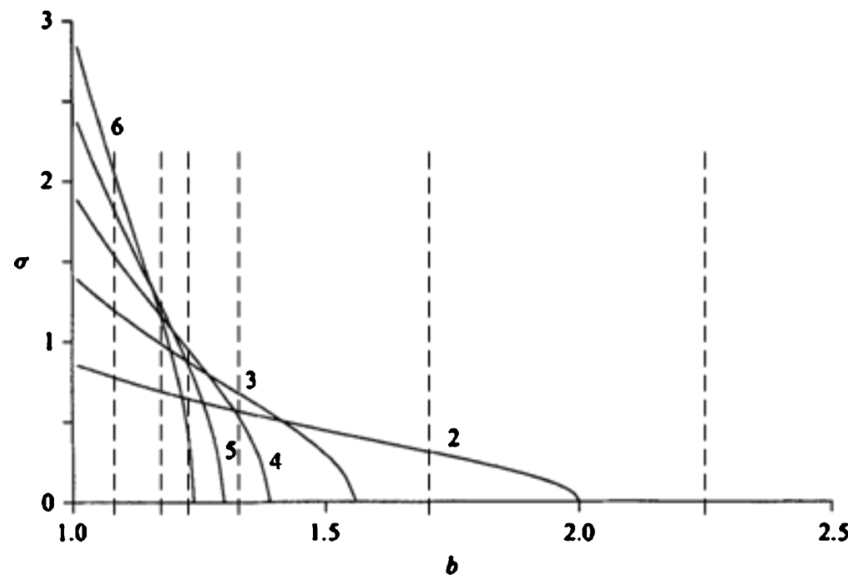

FIG. 1. Growth rates $\sigma$ for barotropic perturbations of an isolated barotropic monopole as a function of the outer radius $b$ for modes $m=2, \ldots, 6$ (after Ref. 2). The dashed lines indicate the values of $b$ used in the experiments presented below (see Table I)

number $m$ of the most unstable azimuthal mode (see Fig. 1). We will exploit this result to generate unstable laboratory vortices whose nonlinear evolution may result in the formation of a multipolar vortex with the number of satellite vortices being equal to the number of the fastest growing mode. Besides tripolar and quadrupolar vortices, the formation of a pentapolar vortex that arises spontaneously from an initially isolated circular vortex will be demonstrated. Also, evidence of the onset of hexapolar and heptapolar vortices was found. The experimentally produced multipoles will be compared with the semianalytical solutions for multipolar vortices obtained by Kizner et al., ${ }^{30}$ with the emphasis put on the form of the vorticity versus streamfunction relation and the crosssectional distribution of vorticity.

The paper is organized as follows. In Sec. II the experimental setup is introduced and the technique for the generation of multipolar vortices is discussed. The nonlinear evolution of unstable monopolar vortices is presented in Sec. III, and the comparison of finite-amplitude flow structures with the semianalytical solutions by Kizner et $a l^{30}$ is given in Sec. IV. A summary of the main results and the conclusions appear in Sec. V.

\section{EXPERIMENTAL SETUP}

The experimental arrangement is shown schematically in Fig. 2. The setup consists of a rectangular perspex tank with dimensions $150 \times 100 \times 30 \mathrm{~cm}^{3}$ which is mounted on top of a rotating table. The tank was filled with water and the flow was allowed to adjust to a solid-body rotation for at least half an hour. In order to obtain a constant fluid layer depth and to avoid topographic production of vorticity, the tank was equipped with a parabolic bottom which compensates for the parabolic free surface. The angular velocity of the rotating tank $\Omega$ was chosen to be $0.7 \mathrm{rad} \mathrm{s}^{-1}$ (which corresponds to a rotation period $T=9 \mathrm{~s}$ ) and the fluid depth $H$ was set to $20 \mathrm{~cm}$.

A circular vortex with zero net circulation was generated by a system of two concentric thin-walled cylinders with radii $R_{1}$ and $R_{2}$ rotating around their vertical axes. Here and

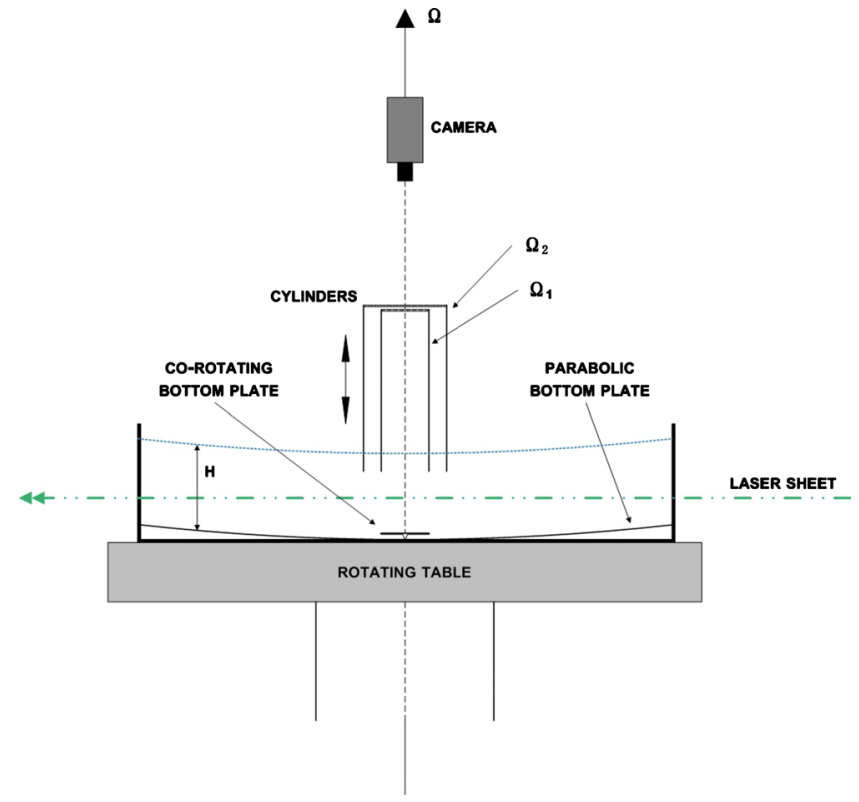

FIG. 2. (Color online) Side view of the experimental setup.

in what follows, indices 1 and 2 refer to the inner and the outer cylinder, respectively. The cylinders were mounted on a translation mechanism allowing the cylinders to be moved in the vertical and the horizontal directions. Each cylinder has a length of approximately $30 \mathrm{~cm}$ and a wall thickness of $0.5 \mathrm{~mm}$. The radius of the outer cylinder was taken constant at $R_{2}=75.0 \mathrm{~mm}$, whereas the radius of the inner cylinder was varied within the range $R_{1}=33.5 \mathrm{~mm}$ to $R_{1}=70.0 \mathrm{~mm}$. In this way the ratio of the outer to the inner cylinder radius, $b=R_{2} / R_{1}$, was effectively changed within the range of $1.07-$ 2.25. In four of the six experiments, the values of $b$ were chosen at the center of the interval within which the growth rate of a specific mode- $m$ perturbation should be the largest compared to other modes (see Fig. 1).

Both cylinders are allowed to rotate independently with angular velocities $\Omega_{1}$ and $\Omega_{2}$, respectively. As the focus of this paper is on isolated vortices, only cases for which $\Omega_{2}=0$ will be presented. The rotation speed of the inner cylinder was set to $\Omega_{1}=0.35 \mathrm{rad} \mathrm{s}^{-1}$, implying a Rossby number $\operatorname{Ro}=\Omega_{1} / 2 \Omega=0.25$, so that the condition of a quasi-twodimensional flow is reasonably satisfied. For much lower values of $\Omega_{1}$ the linear stage of instability growth could not be captured owing to the relatively large amplitudes of the disturbances induced by the lifting of the cylinders (see below). Important experimental parameters are summarized in Table I.

In order to obtain a perfect solid-body rotation within the inner cylinder, the latter was equipped with a detachable bottom disk rotating along with the inner cylinder. The disk had the same radius as the inner cylinder and was fixed to the parabolic bottom by a short axle. The thickness of the disk $(0.25 \mathrm{~mm})$ could be regarded as negligible relative to the depth of the fluid. The rotation of the inner cylinder was transmitted to the disk by friction when the cylinder was at its lowest vertical position. Without a corotating bottom disk, some vorticity anomaly (different from the desired) would 
TABLE I. Overview of different cylinder configurations. Here, $m$ indicates the theoretically most unstable wave number for the piecewise-uniform model vortex (Ref. 2), $R_{1}$ and $R_{2}$ are the radii of the inner and outer cylinders, $b=R_{2} / R_{1}$ is the ratio of cylinder radii, and $\Omega_{1}$ and $\Omega_{2}$ are the corresponding angular velocities.

\begin{tabular}{cccccc}
\hline \hline & $\begin{array}{c}R_{1} \\
(\mathrm{~mm})\end{array}$ & $\begin{array}{c}R_{2} \\
(\mathrm{~mm})\end{array}$ & $\begin{array}{c}b \\
(-)\end{array}$ & $\begin{array}{c}\Omega_{1} \\
\left(\mathrm{rad} \mathrm{s}^{-1}\right)\end{array}$ & $\begin{array}{c}\Omega_{2} \\
\left(\mathrm{rad} \mathrm{s}^{-1}\right)\end{array}$ \\
\hline 1 & 33.5 & 75.0 & 2.25 & 0.35 & 0 \\
2 & 44.0 & 75.0 & 1.70 & 0.35 & 0 \\
3 & 56.0 & 75.0 & 1.34 & 0.35 & 0 \\
4 & 61.0 & 75.0 & 1.23 & 0.35 & 0 \\
5 & 63.5 & 75.0 & 1.18 & 0.35 & 0 \\
6 & 70.0 & 75.0 & 1.07 & 0.35 & 0 \\
\hline \hline
\end{tabular}

have been developing only within the Stewartson boundary layers $^{32}$ which reside along the inner and outer walls of the inner cylinder and along the inner wall of the outer cylinder. These boundary layers have a thickness of typically $H E^{1 / 4}$, with $E=\nu /\left(\Omega H^{2}\right)$ the Ekman number. With the current parameter settings the thickness of the Stewartson boundary layers is only $1-2 \mathrm{~cm}$ so that the preferred initial condition cannot be obtained with the open inner cylinder. The presence of the corotating bottom disk allows the fluid inside the inner cylinders to spin up to solid-body rotation by Ekman suction within a time period of typically $T_{E}=H /(\nu \Omega)^{1 / 2}$ $=240 \mathrm{~s}$ (generally referred to as the Ekman spin-up time). For practical reasons, no corotating bottom was used for the annulus region between the two cylinders, but it can be shown analytically that the vorticity distribution in the annulus region is close to the desired value of opposite-signed uniform vorticity, the more so for smaller values of $b$.

Below, the procedure is described that was followed to generate, visualize, and monitor the vortex. When the fluid in the rectangular tank was at rest (relative to the rotating frame of reference) the inner and outer cylinders were gently lowered into the fluid until they touched the bottom disk and the parabolic bottom, respectively. The inner cylinder was set into motion and after an adaptation period of approximately half an hour, the flow inside the cylinders was stationary and both cylinders were lifted within half a rotation period of the rotating table without lifting the bottom disk. The time at which the cylinders are lifted out of the fluid will be defined as $t=0$. Finally, the cylinders were moved aside so as to be able to view the motion from above.

In order to obtain qualitative information about the flow evolution, the fluid was visualized with different colors of dye before the cylinders were withdrawn. The region inside the inner cylinder was injected with "terasil brilliant rosa" (red) whereas the annulus was injected with "fluorescein" (green). The flow was illuminated with ordinary strip lighting and recorded from above with a color CCD camera with a resolution of $720 \times 576$ pixels and a frame rate of $25 \mathrm{~Hz}$. In order to obtain quantitative data, the flow was seeded with passive tracers (polystyrene particles) with a diameter of $50 \mu \mathrm{m}$ and with a mean density virtually equal to that of water at room temperature. The tracers were illuminated at mid depth by a laser sheet (Fig. 2) which originated from a double pulsed Nd:YAG laser. The tracers were monitored from above by a 10-bit gray level CCD camera with a resolution of $1008 \times 1019$ pixels and a frame rate of $15 \mathrm{~Hz}$. Image pairs were cross-correlated with the technique of particle image velocimetry (PIV) from PIVTEC GmbH (Göttingen, Germany) to retrieve the horizontal velocity field. Depending on average spatial particle density, either interrogation areas of $16 \times 16$ pixels or $24 \times 24$ pixels were used. The vorticity and streamfunction were computed by numerical differentiation and integration of the horizontal velocity field, respectively.

\section{NONLINEAR EVOLUTION}

Figure 3 shows the measured cross-sectional distributions of the azimuthal velocity and the (computed) vorticity for the monopolar vortex just after the lifting of the cylinders at $t=T$ (solid lines). The cross-section was taken along an arbitrary line through the vortex center $r=0$, where $r$ param-
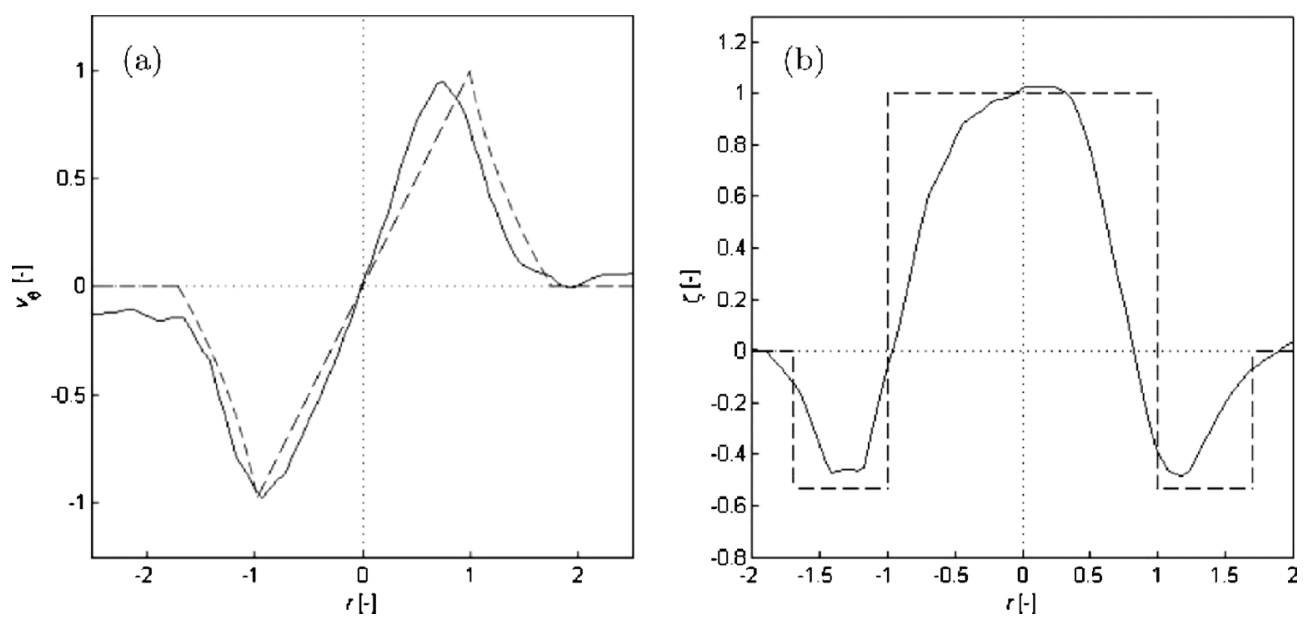

FIG. 3. Cross-sectional distributions of (a) the azimuthal velocity and (b) the vorticity of an isolated monopolar vortex generated by a pair of cylinders with diameter ratio $b=1.70$. The solid lines correspond to the profiles of the observed monopolar vortex at $t=T$. The dashed lines correspond to the desired profiles of a piecewise-uniform monopolar model vortex. The velocity and the vorticity have been normalized with the maximum values associated with the isolated monopolar model vortex. The scaled radial coordinate $r(-)$ (can be positive and negative) has been scaled with the radius of the inner cylinder. 
etrizes the position at the line (that is, can be both positive and negative). Also shown are the corresponding profiles of the desired "model vortex" (dashed lines) which consists of a core of uniform vorticity and a ring of uniform but oppositesigned vorticity and has zero total circulation. Both the azimuthal velocity and the vorticity have been scaled with the maximum velocity and the maximum vorticity associated with the model vortex. The coordinate $r$ has been scaled with the radius of the inner cylinder, $R_{1}$.

Owing to the three-dimensional turbulent mixing induced by the lifting of the cylinders, and also due to the lateral diffusion of vorticity, the measured vorticity distribution is not piecewise-uniform. Nevertheless, the peak values of the measured vorticity are in excellent agreement with the corresponding vorticity values of the model vortex. Moreover, the locations of zero vorticity are close to the original positions of the cylinder walls, and the measured profile of azimuthal velocity reveals that the finite vorticity gradients cause relatively small deviations from the model profile. Thus, our technique allows a quite precise control of the relative width of the annulus.

The profiles shown in Fig. 3 are typical of the initial stages of monopolar vortices (either stable or unstable) up to wavenumber-4 perturbations. For monopolar vortices which were unstable to higher-order modes, the vortex core was observed to be subject to strong inertial oscillations. This issue will be discussed later on in Sec. III. In all cases, the absolute value of the initial ratio of positive and negative circulations was $1.00 \pm 0.05$.

In Figs. 4 and 5, examples of the evolution of dyevisualized monopolar vortices are shown. The linear stage of the development of perturbations growing on these monopoles is expected to be dominated by wavenumber $m=2,3$, 4 , and 5 perturbations, respectively. Figure 4(a) demonstrates the evolution of an isolated monopolar vortex that was generated by a set of cylinders with the diameter ratio $b=1.70$. Times are indicated in the lower-right corner of each frame, with $T$ as the rotation period of the turntable. During the initial stage of the evolution, both the vortex core and the annulus are well defined and virtually axisymmetric. After a few rotation periods $T$, however, the shape of the vortex core becomes slightly elliptical which reveals the linear growth of a wavenumber- 2 perturbation. When time progresses, the asymmetry of the vortex core becomes more conspicuous, and the annulus eventually rearranges into two pronounced satellite vortices. In contrast with previous rotating tank experiments ${ }^{13-16}$ in which the nonlinear evolution of wavenumber-2 perturbations resulted in a persistent tripolar vortex structure, the present experiment reveals a different evolution scenario. As evident from the arrangement of the satellite vortices at $t=6.0 T$ and subsequent times, the rotation rate of the elliptically shaped vortex core does not match the azimuthal propagation speeds of the satellite vortices. As a result, the vortex core is torn apart and the vortex tends to break up into two dipolar vortices that move in opposite directions away from the original vortex center. The observed nonlinear evolution is in close agreement with numerical simulations by Flierl ${ }^{2}$ and Morel and Carton ${ }^{9}$ who showed a similar breakup of monopolar vortices with a piecewise-uniform vorticity distribution using the technique of contour dynamics. ${ }^{31}$ The fact that the current results deviate from those obtained in previous rotating tank experiments is probably related to the different forcing techniques used. The stirring technique introduced by Van Heijst and Kloosterziel $^{12}$ (see also Ref. 14) leads to a smoother vorticity profile than that obtained in the present study, which may explain the different nonlinear evolutions scenarios. Indeed, pseudospectral simulations ${ }^{3,7}$ revealed the formation of a persistent tripolar structure from an initially axisymmetric unstable monopolar vortex with distributed vorticity. In this respect, we do not exclude the possibility of the production of stable tripoles for other values of $b$ within the range $1.55<b<2$, especially for values of $b$ larger than $b=1.70$ (i.e., smoother vorticity profiles), but in the present study this range of values was not explored in detail.

In Fig. 4(b), the evolution of a monopolar vortex is shown with a narrower ring of opposite-signed vorticity, $b=1.34$, than in the previous example $(b=1.70)$. The same colors of dye were used to mark the core and the annulus of the initially axisymmetric monopolar vortex. Following the analytical study by Flierl ${ }^{2}$ this vortex is expected to be most unstable to wavenumber-3 perturbations. Indeed, already in the very early stage of the evolution, at $t=2.0 T$, a wavenumber-3 instability develops, which is apparent in the deformation of the core and the mode- 3 wave pattern in the annulus. Soon thereafter, at $t=3.0 T$ and onward, the nonlinear phase of the vortex evolution manifests itself by a major rearrangement of the dye distribution resulting in a quadrupolar vortex structure which is characterized by a pronounced triangular-shaped core and three distinctive satellite vortices. Note that a small amount of fluid originating from the vortex core entrains into the satellite vortices. The motion in the vortex core is cyclonic, whereas anticyclonic motion occurs in the satellite vortices. The resulting vortex structure is persistent for about four rotation periods $T$ and rotates in a solid-body-like fashion relative to the rotating frame of reference in cyclonic direction. The quadrupolar vortex turns out to be unstable, as evident from the onset of the merger of two of the three satellite vortices, and eventually tends to degrade into a tripolarlike vortex.

The observed transition of the quadrupolar to a tripolarlike vortex is supported by the normal-mode analysis of Flierl ${ }^{2}$ in which it was shown that axisymmetric vortices with a narrow ring of opposite-signed vorticity are unstable to more than one mode. Indeed, besides being unstable to wavenumber- 3 perturbations, it may be inferred from Fig. 1 that the present initial monopolar vortex is also unstable to wavenumber-2, and to a lesser extent to wavenumber-4 perturbations (assuming an initial piecewise-uniform distribution of vorticity). The wavenumber-2 instability manifests itself somewhat later due the corresponding smaller growth rate. It should be noted, however, that in principle this simple argument does only hold for the linear stage of the evolution of the monopolar vortex. More solid support for the degeneration of multipolar vortex structures into structures of lesser complexity was given analytically by Morel and Carton, ${ }^{9}$ who performed an instability study for a multipolar vortex composed of a central core vortex with uniform vor- 


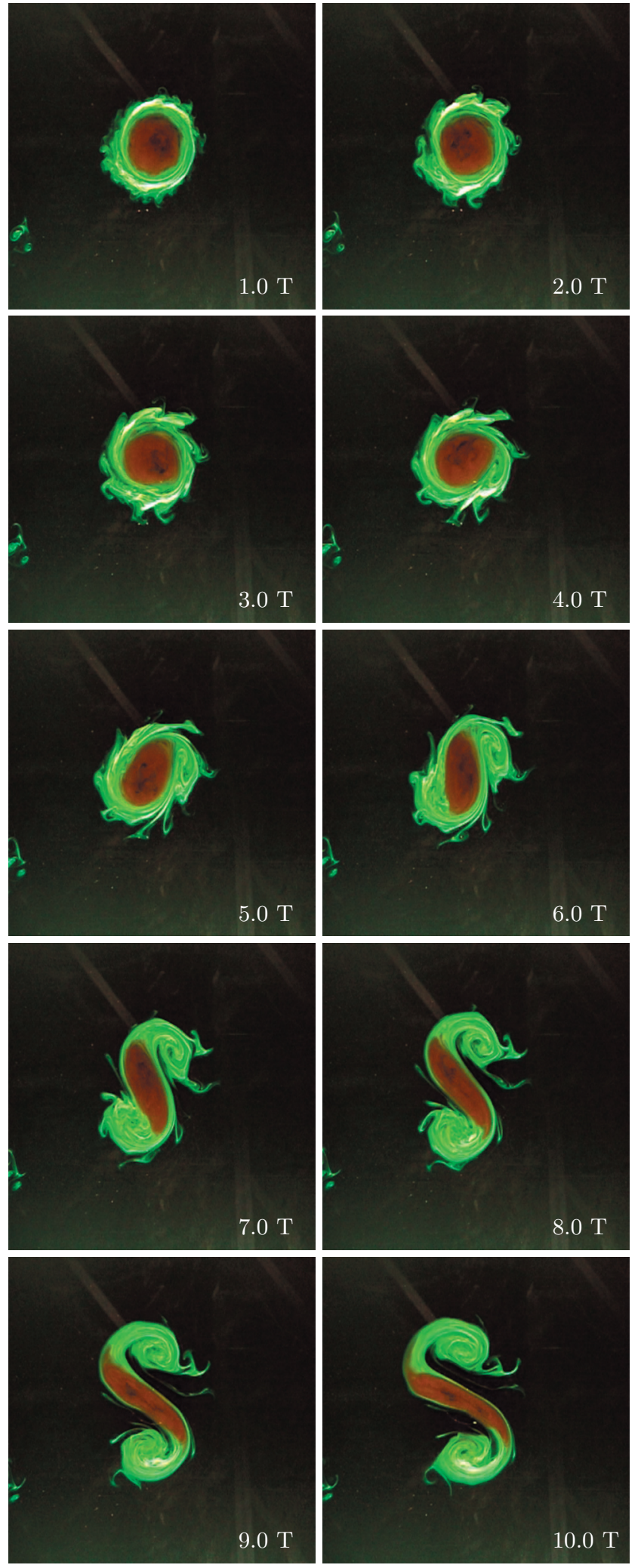

(a)
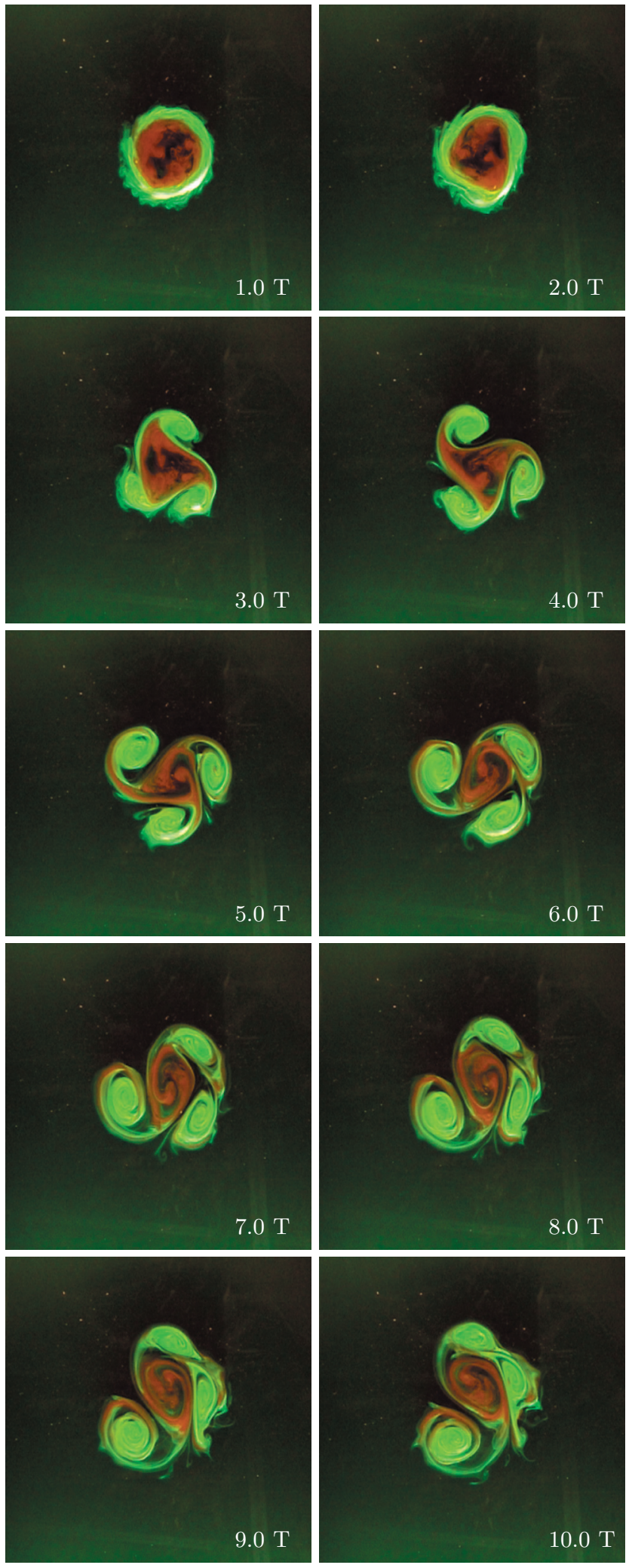

(b)

FIG. 4. (Color) Dye-visualized evolution of an isolated monopolar vortex generated by a set of cylinders with diameter ratios (a) $b=1.70$ and (b) $b=1.34$, respectively. The regions inside the inner cylinder and the annulus were injected with red and green dyes, respectively. Times are indicated in the lower-right corner of each frame, with $T$ as the rotation period of the turntable.

ticity and $m$ satellite point vortices. It was shown that multipoles with an even number of satellites mostly degenerate into twice as simple structures, whereas multipoles with an odd number of satellites undergo an asymmetric transition like the one shown in Fig. 4(b). Here it should be kept in mind, though, that the finite sizes of the satellite vortices were neglected in the analytical study by Morel and Carton ${ }^{9}$ so that the effects of merger events were not taken into ac- 


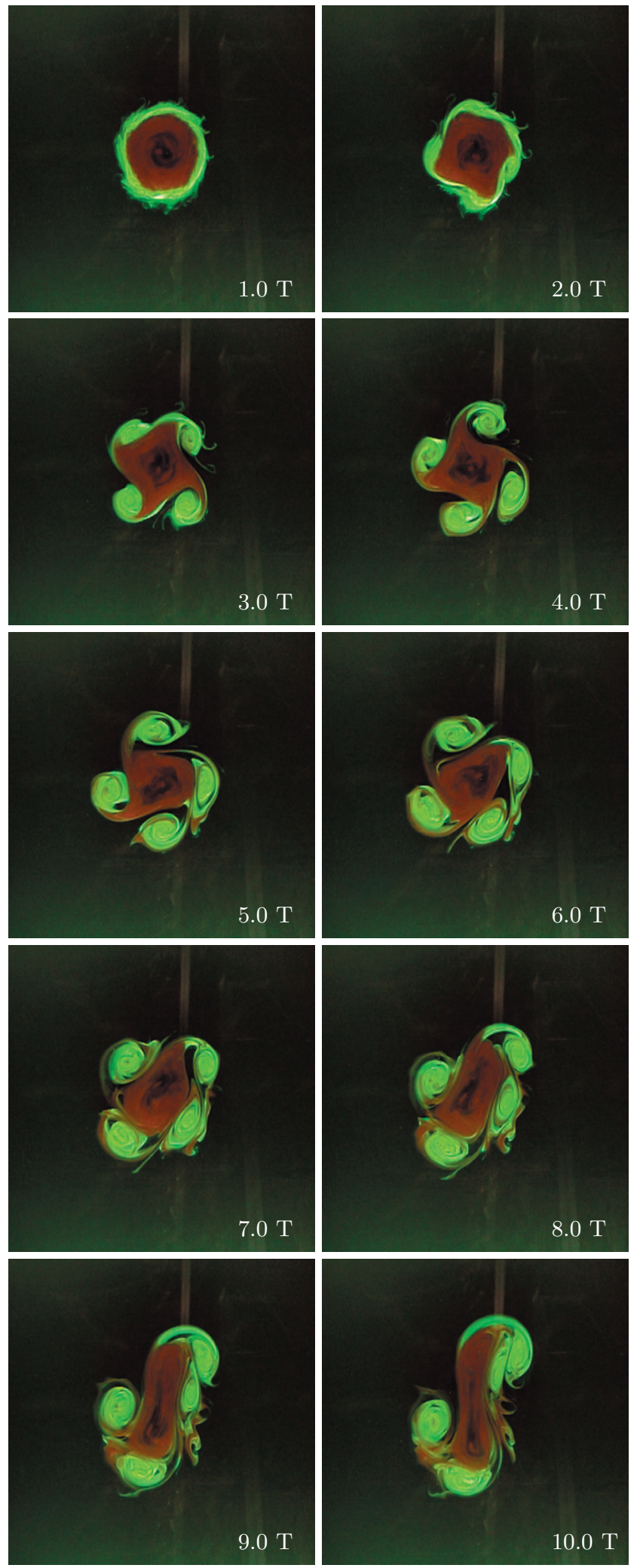

(a)
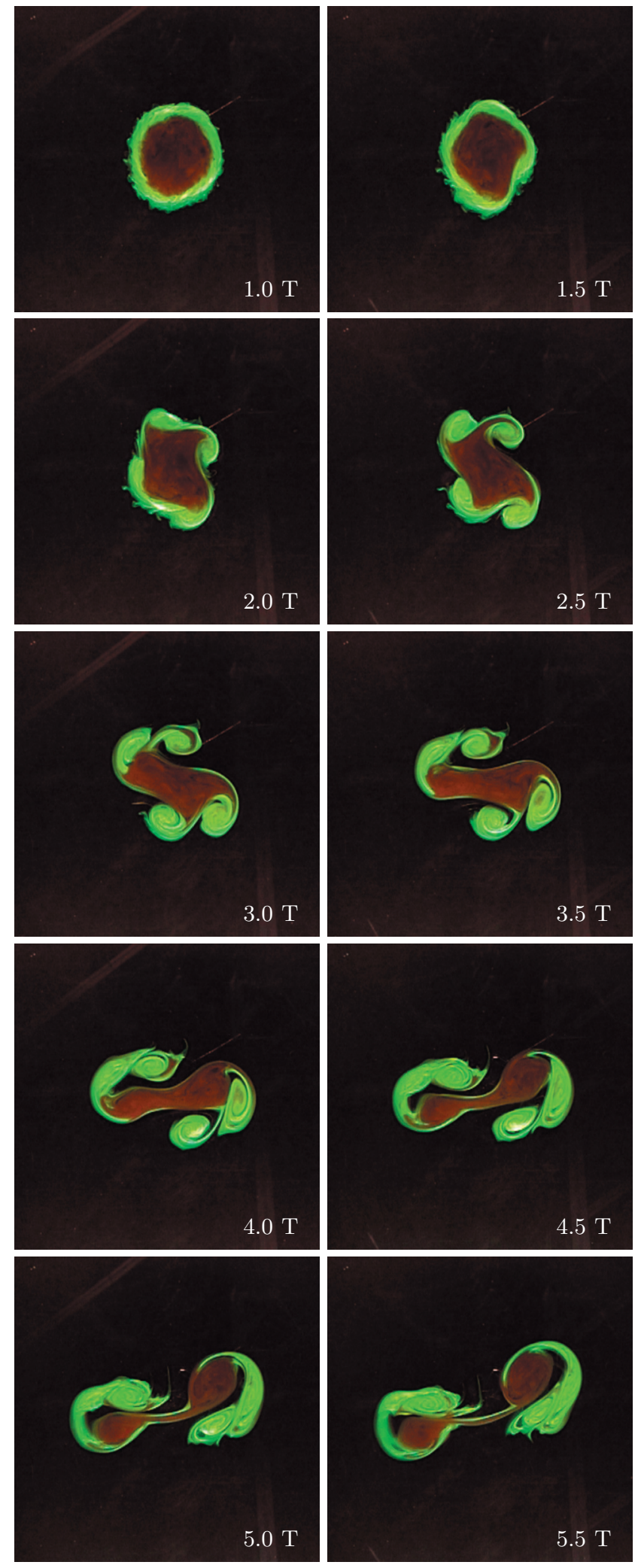

(b)

FIG. 5. (Color) Same as Fig. 4, but with diameter ratios (a) $b=1.23$ and (b) $b=1.18$, respectively.

count. The quadrupole-to-tripole degradation is in agreement with the inverse energy cascade concept, because in this transition, three relatively small satellite vortices reorganize into a pair of bigger satellites (see also Sec. V, where the evolution of higher-order multipoles is discussed).
Motivated by the observation of the quadrupolar vortex, we have searched for even higher-mode multipolar vortices by further decreasing the width of the initial ring of oppositesigned vorticity. Figure 5(a) shows the dye-visualized evolution of an isolated monopolar vortex generated by a set of 
cylinders with diameter ratio $b=1.23$. According to the normal-mode calculations of Flierl $^{2}$ the corresponding monopolar vortex is most unstable to wavenumber-4 perturbations and may evolve into a pentapolar vortex. Figure 5(a) demonstrates that the wavenumber- 4 instability indeed prevails during the initial stage of the evolution of such a vortex. During the nonlinear phase of the instability, the vortex core acquires the shape of a square, whereas the annulus rearranges into four well-defined satellite vortices. As far as the authors are aware, this is the first laboratory observation of a pentapolar vortex that arises spontaneously from an initially isolated circular vortex. The pentapolar vortex has characteristics similar to those of the quadrupolar vortex shown in Fig. 4(b). This similarity applies to the flow direction both in the core and in the satellite vortices as well as to the rotation of the entire vortex structure. Note again the entrainment of core fluid into the satellite vortices. Moreover, the pentapolar vortex also turns out to be unstable, which is observed from the merger of two of the four satellite vortices. During this stage, the evolution seems to be dominated by a wavenumber-3 perturbation-consistent with the analytical results of Flierl, ${ }^{2}$ see Fig. 1-but in a later stage the perturbed pentapolar vortex tends to break up into two dipolar vortices, as predicted by the simple point-vortex based model of Morel and Carton. ${ }^{9}$

As a final example, we present the evolution of a monopolar vortex that was generated by a set of cylinders with a diameter ratio $b$ of only 1.18 . Figure 1 reveals that for this configuration the normal-mode calculations predict the fastest growth rate for wavenumber-5 perturbations. Note, however, that the growth rate of wavenumber-4 perturbations is very close to that of wavenumber- 5 perturbations. Figure 5(b) shows the evolution of the monopolar vortex with $b=1.18$. During the initial stage, one may identify a weak wavenumber-5 perturbation both in the core and in the annulus, but soon thereafter, the vortex evolves into a highly unstable pentapolar vortex which breaks up into two dipolar vortices. For each dipolar vortex, the cyclonic part originates from the original vortex core, whereas the anticyclonic part is the result of the merger of two neighboring satellite vortices.

In Figs. 6-9 we show the time evolution of the vorticity and the horizontal velocity fields for the same initial flow configurations presented in Figs. 4 and 5. The areas of positive and negative vorticity are indicated by the shading, where darker shading corresponds to higher magnitudes of vorticity. In order to emphasize the regions of positive and negative vorticity, we normalized the vorticity values by the maximum vorticity value at the corresponding time. In addition, we used a nonlinear relationship between the shading intensity and the vorticity magnitude. As a consequence, dark-shaded regions correspond to a much wider range of vorticity amplitudes than one would expect from a linear relationship. The horizontal velocity field is indicated by the arrows. The circles in the first panel mark the original positions of the cylinders. Times are indicated below each panel, scaled with $T$, the rotation period of the turntable.

It should be stressed that both the vorticity and the horizontal velocity were obtained from a separate set of experi-

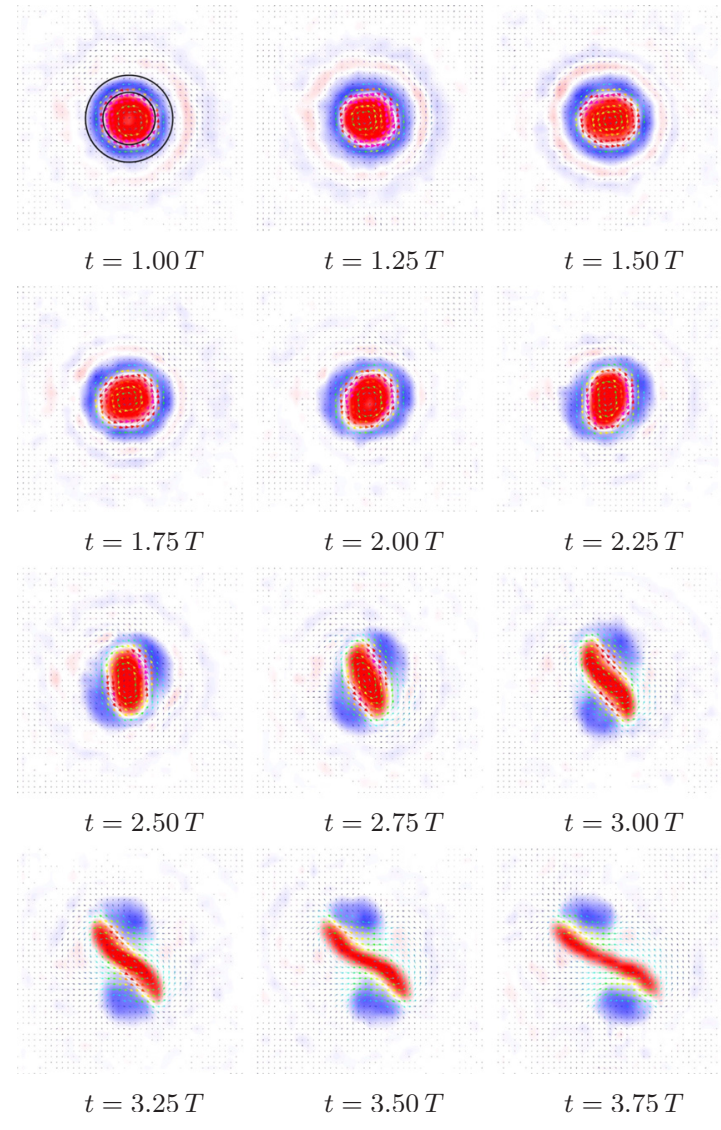

FIG. 6. (Color online) Time-evolution of the vorticity and the horizontal velocity fields for an isolated monopolar vortex generated by a pair of cylinders with diameter ratio $b=1.70$. The regions of positive and negative vorticity (located, respectively, in the central and peripheral parts of each frame) are shaded, whereas the horizontal velocity field is indicated by the arrows. The circles in the first panel mark the original positions of the cylinders. Times are indicated below each panel, with $T$ the rotation period of the turntable.

ments so that the details of the different flow scenarios as well as the times of the different stages of the evolution may differ. Concerning the timing of the PIV experiments, it was observed that the development of the instabilities and the subsequent nonlinear evolution was generally faster than in the dye experiments. The faster growth of instabilities may be attributed to the presence of external perturbations induced by the pumping system of the corotating laser-cooling device. We also note that the magnitudes of both the vorticity and the velocity decrease exponentially in time due to the presence of Ekman boundary layers at the bottom of the rotating tank. Since the nonlinear stage in the PIV experiments sets in sooner, the subsequent development is also faster than in the dye experiments.

Figure 6 shows the development of an unstable monopolar vortex in terms of the vorticity and the horizontal velocity field for the case $b=1.70$. As shown before, the monopolar vortex is subject to a wavenumber-2 instability which leads to the formation of an elliptically shaped vortex core and two satellite vortices. Note that the regions of positive and negative vorticity are well separated: the vortex core is almost entirely made up of positive vorticity, whereas the fluid inside the satellite vortices has only negative vorticity. In the 


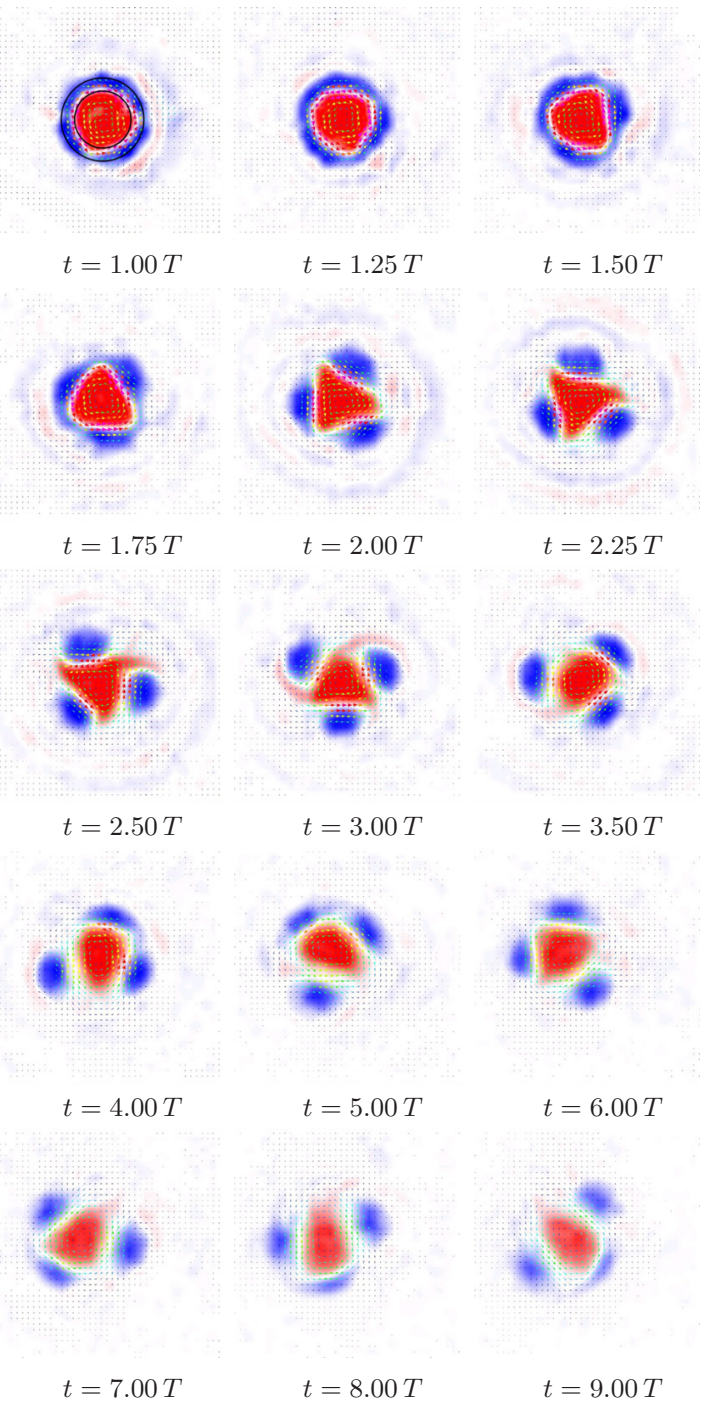

FIG. 7. (Color online) Same as in Fig. 6, but at $b=1.34$.

final stage of the evolution, the vortex core is torn apart and the vortex tends to break up into two dipolar vortices that move in opposite directions away from the original vortex center, much in the same way as shown in the corresponding dye experiment presented in Fig. 4(a).

During the initial stage of the flow evolution, we also observe rings of alternating positive and negative vorticity, which are most noticeable in the region exterior to the vortex. Close inspection of the first few panels in Fig. 6 reveals that these rings move inward toward the vortex center, while their magnitude gradually decreases in time. (The rings are seemingly more persistent owing to the normalization of the vorticity with the decaying maximum vorticity.) Although at present, the dynamics of these rings is not completely understood, we believe that they are related to inertial oscillations that are excited by the lifting of the cylinders (note that the initial value of the Rossby number, $\mathrm{Ro}=0.25$, though small, still allows some rotating-shallow-water effects at the very beginning of the experiment). This point of view is supported by a spectral analysis which shows that the frequency of these oscillations is close to $2 \Omega$.

The development of the vorticity field of a quadrupolar

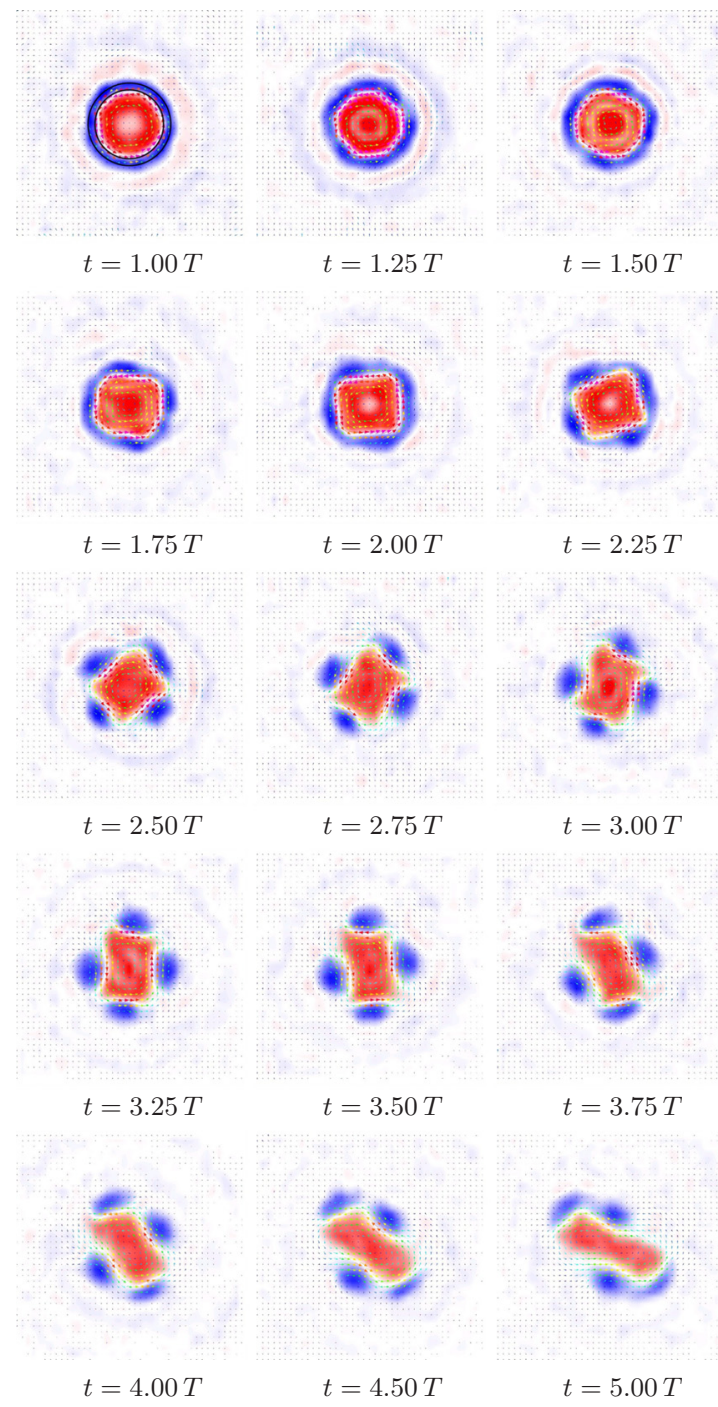

FIG. 8. (Color online) Same as in Fig. 6, but at $b=1.23$.

vortex is presented in Fig. 7 for the case $b=1.43$. Apart from the timing, the process is very similar to that shown in Fig. 4(b). The similarity is apparent in the formation of a triangular-shaped vortex core, the rearrangement of the annulus into three satellite vortices, and the overall rotation of the quadrupolar vortex around its center. Also note again the entrainment of core fluid into the satellite vortices, which is evident from the vorticity filaments emanating at the corners of the triangular-shaped vortex core. The quadrupolar vortex is unstable and finally transforms into a tripolarlike vortex. Note that the merger of two of the three satellite vortices is prematurely ended (see $t=4.00 T-6.00 T$ ) and that, in contrast with the dye-visualized evolution in Fig. 4(b), the tripolar vortex results from the shearing out of one of the satellite vortices (see $t=7.00 T-9.00 T$ ).

Figure 8 shows the vorticity and velocity fields associated with the development of the pentapolar vortex for the case $b=1.23$. The pentapolar vortex is unstable and tends to break up into two quite symmetrical vortex pairs (dipoles) which move away from each other. This scenario is in close agreement with that observed for dye-visualized vortices [Fig. 5(a)]. In addition, the vorticity distributions reveal a 


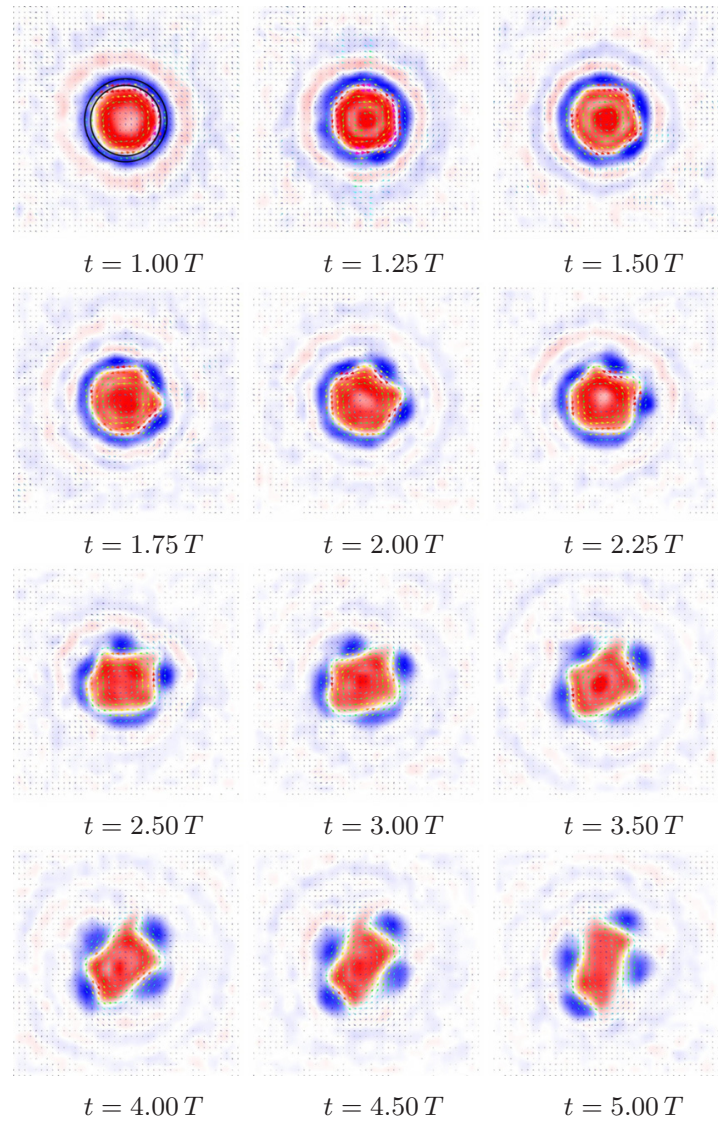

FIG. 9. (Color online) Same as in Fig. 6, but at $b=1.18$.

phenomenon which was not visible in the dye experiments. We observe that the magnitude of the vorticity in the vortex core changes significantly in a periodic fashion. Remarkably, such vigorous oscillations were not observed in the vorticity distributions related to multipolar vortices of lower order.

In Fig. 9 we show the evolution of the monopolar vortex for the case $b=1.18$. During the initial stage, we observe a weak wavenumber-5 perturbation, which is apparent in the polygonal shape of the vortex core and the tendency of the annulus to reorganize itself into five satellite vortices. The recognition of a possible hexapolar vortex is complicated by the fact that satellite vortices become progressively smaller for higher-order multipoles. ${ }^{9}$ Even so, evidence was found also of the onset of a heptapolar vortex for the case $b$ $=1.07$ (not shown). Eventually, however, only four satellite vortices survive, leading to the formation of a highly unstable pentapolar vortex whose further development is similar to that shown previously in Fig. 5(b). Also in this case, we observe that the vorticity in the vortex core changes vigorously in a periodic fashion. The fact that the frequency of oscillation is close to $2 \Omega$ suggests that the oscillations may have the same origin as the vorticity rings of alternating sign.

The origin of the high-amplitude oscillations in the vortex core may be explained by the results of a recent analytical/numerical study ${ }^{33}$ in which it was shown that for the case of a confined cyclonic vortex with background rotation the local frequency of the inertial oscillations ranges from zero up to the value of the local absolute vorticity (i.e., $2 \Omega+\zeta)$. In our case, the annulus of opposite-signed (relative)
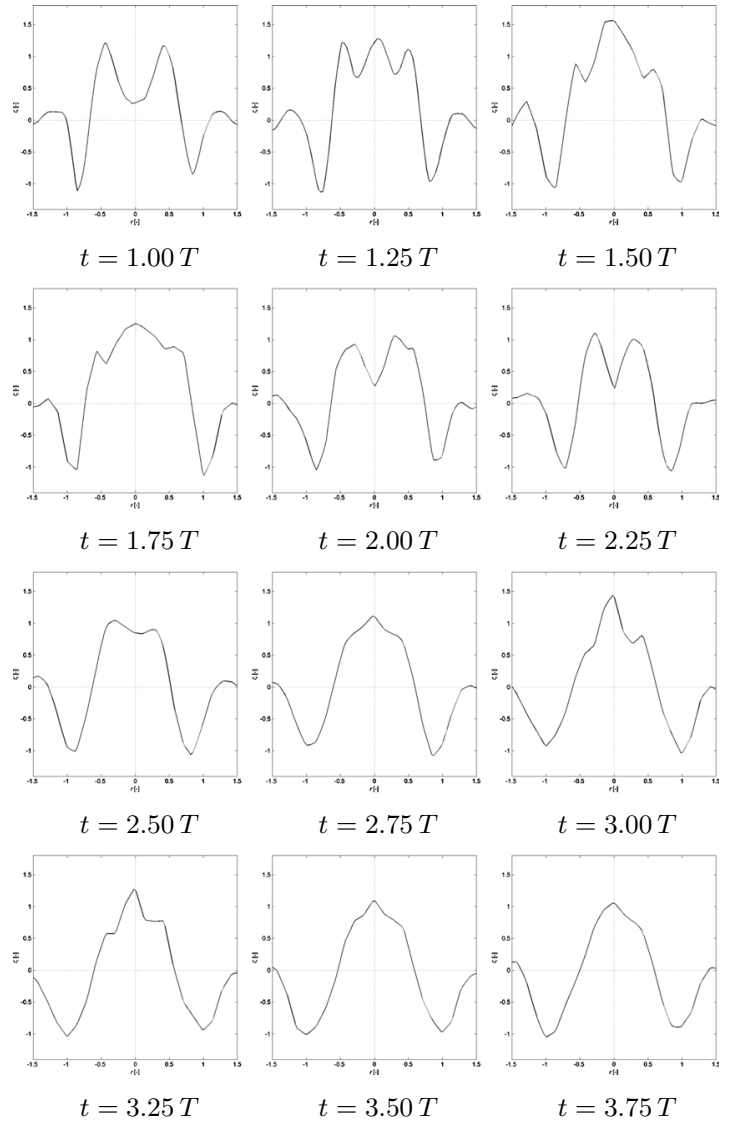

FIG. 10. Cross-sectional vorticity distributions along a straight line passing through the vortex center and possibly through one of the (developing) satellite vortices for the vortex shown in Fig. 8. Normalization as in Fig. 3.

vorticity $\zeta$ contains relatively low absolute vorticity $2 \Omega+\zeta$ which may prevent the core oscillations to radiate radially outward. This "barrier effect" is expected to be more efficient for vortices with a narrower shield of opposite-signed (relative) vorticity (up to the point where the absolute vorticity in the annulus becomes zero). The time series of vorticity profiles for the experimental pentapolar vortex, see Fig. 10, confirm that the oscillations are generally concentrated in the core and are virtually absent in the ring of opposite-signed relative vorticity. Moreover, the above explanation is supported by the observation that the oscillations in the core are more dominant for monopolar vortices with a narrower shield of opposite-signed (relative) vorticity.

One may argue that the initial decrease of vorticity in the vortex core is due to strong Ekman pumping. However, numerical simulations of the evolution of an axisymmetric vortex with an initially piecewise-uniform vorticity distribution demonstrated that even for vortices with a very narrow ring of opposite-signed relative vorticity $(b=1.07)$ Ekman pumping cannot account for a decrease of more than $10 \%$ of the initial vorticity in the vortex center within one rotation period. The current experiments, though, show a decrease of more than $50 \%$ during the same time period (see for example the first panel of Fig. 10). The discrepancy may be due to strong perturbations induced by the lifting of the cylinders, which are not accounted for in the numerical simulations. 

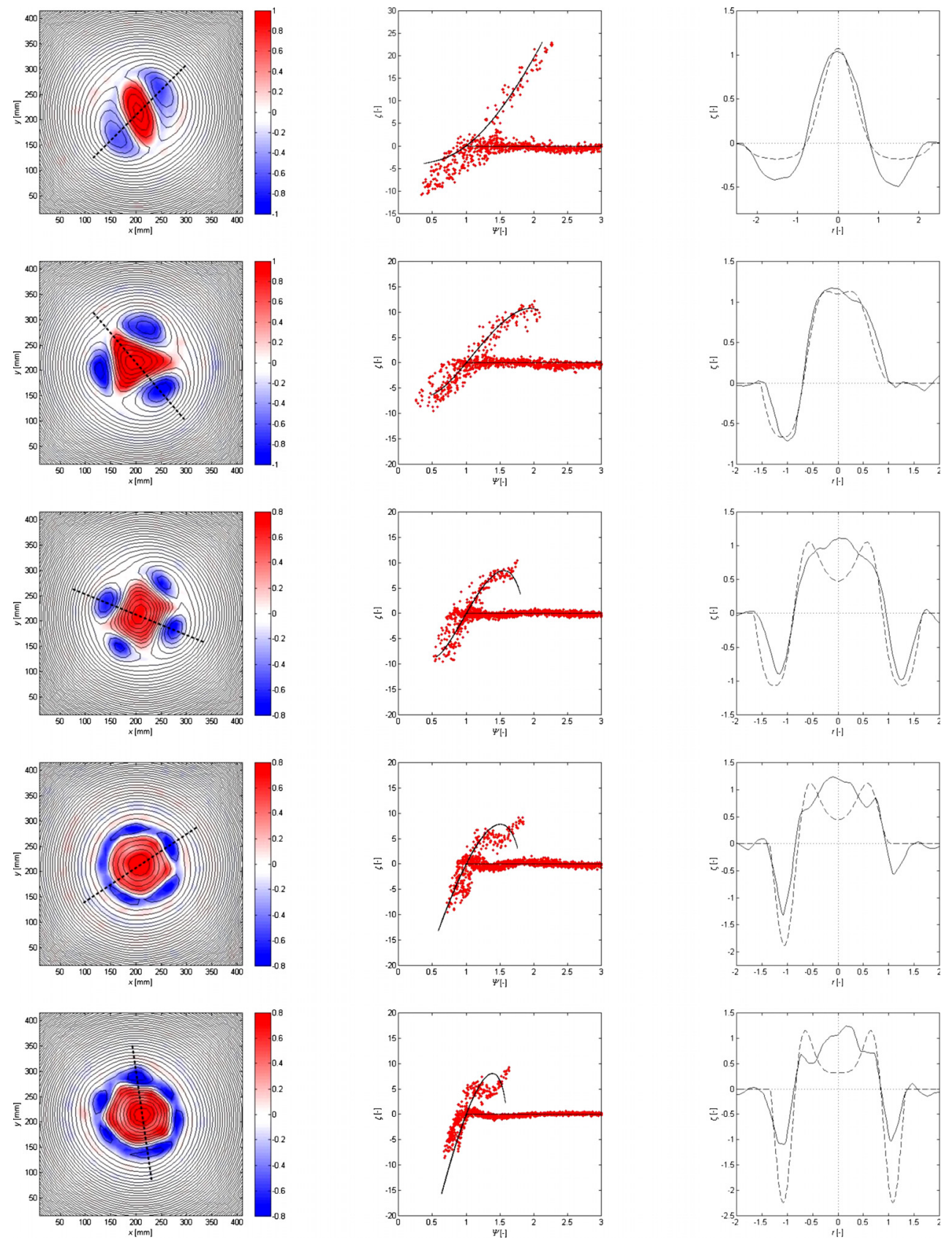

FIG. 11. (Color online) Comparison of experimental results with the semianalytical solutions for multipolar vortices by Kizner $e t$ al. (Ref. 30). Left panels: distributions of vorticity (colored regions) and corotating streamfunction (contours). Middle panels: vorticity vs corotating streamfunction relationships. Right panels: cross-sectional vorticity distributions along a straight line (dashed lines in the left panels) passing through both the center of the vortex core and the center of one of the satellite vortices. The solid lines in the middle panels and the dashed lines in the right panels indicate the semianalytical solutions by Kizner et al. (Ref. 30). The vorticity in the middle panels has been normalized with the angular velocity of the vortex structure relative to the rotating frame of reference, $\Omega_{v}$, whereas the corotating streamfunction has been scaled with the average value of the corotating streamfunction at the separatrix, $\Psi_{s}$. Both the vorticity and the distance along the cross-section have been normalized as in Fig. 3. The experimental data were selected from the experiments presented in Figs. 6-9 and correspond to the following times, respectively: $t=2.7 T, t=1.9 T, t=2.8 T, t=1.6 T$, and $t=1.3 T$.

\section{MULTIPOLAR VORTEX CHARACTERISTICS}

We now compare the experimental results presented in the previous section with the semianalytical solutions for multipolar vortices obtained by Kizner et al. ${ }^{30}$ Figure 11 shows selected data obtained from the experiments presented in Figs. 6-9. For each experiment the most symmetrical vorticity distribution was chosen, although for the tripolar vortex a compromise was made between a symmetric vorticity distribution and a well-developed set of satellite vortices.
The left panels of Fig. 11 show the selected distributions of vorticity $\zeta$ (shaded regions) along with the streamfunction in the frame corotating with the satellite vortices, $\Psi$ (contours). The streamfunction $\Psi$ will be referred to as the corotating streamfunction (not to be confused with the streamfunction $\psi$ in the frame corotating with the water tank, which vanishes at the periphery of the multipole). The middle panels show the corresponding vorticity versus corotating streamfunction relationships. The right panels show the corresponding cross- 
sectional vorticity distribution along a straight line passing through both the center of the vortex core and the center of one or two of the satellite vortices. The cross-sections were taken along the dashed lines indicated in the left panels. The solid lines in the middle panels and the dashed lines in the right panels indicate the semianalytical solutions by Kizner et $a l .{ }^{30}$ The vorticity has been normalized with the angular velocity of the vortex structure relative to the rotating frame of reference, $\Omega_{v}$, whereas the corotating streamfunction has been scaled with the value of the corotating streamfunction at the separatrix, $\Psi_{s}$, which demarcates the region of positive and negative vorticity. Both the vorticity and the distance along the cross-section have been normalized as in Fig. 3 . The angular velocity of the vortex structure was obtained from the average displacement of the satellite vortices during a small time interval around the time corresponding to the specific vorticity distribution. Hence, the corotating streamfunction $\Psi$ follows from the relationship $\Psi=\psi+(1 / 2) \Omega_{v} r^{2}$, where $\psi$ is the streamfunction in the frame corotating with the water tank. In order to determine $\Psi_{s}$, we took advantage of the fact that for a symmetrical vortex structure the stagnation points of the corotating flow lie on the separatrix. The value of $\Psi_{s}$ was obtained from the average of the $\Psi$-values at the stagnation points.

For each case, the corotating streamfunction is closely related to the vorticity. Indeed, the vortex cores and most of the satellite vortices are marked by closed streamlines. This relationship is confirmed by the corresponding vorticity versus corotating streamfunction relationships. Each data point $(\Psi, \zeta)$ corresponds to one of the points of the mesh on which both the vorticity and the corotating streamfunction were computed. The point scatter suggests that the vortex is not completely stationary in the corotating frame of reference, which is evident from the fact that the selected vortices undergo fast inertial oscillations and relatively fast form transformations. Part of the scatter though may be attributed to measurement errors. The scatter appears to be smaller for higher-order multipolar vortices, but this is merely a result of the scaling. Branches of positive vorticity are associated with the vortex cores, whereas branches of negative vorticity are related to the satellite vortices. The horizontal branch around $\zeta=0$ corresponds to the region outside of the vortex.

The agreement with the analytical solutions of Kizner et al. ${ }^{30}$ is fair for the core of the measured tripolarlike vortex, which is also evident from the cross-sectional distribution of vorticity (upper right panel of Fig. 11). For the satellite vortices, however, no good correspondence is obtained. The observed satellites are confined to a smaller areal region and therefore their vorticity magnitudes are larger than those computed by Kizner et al. ${ }^{30}$ Moreover, as noticed earlier, the rotation rate of the elliptically shaped vortex core does not match the azimuthal propagation speeds of the satellite vortices, which explains the large amount of scatter in the branch of negative vorticity and the disagreement with the steady semianalytical solution. A much better and almost excellent agreement is obtained between the experimental data of the quadrupolar vortex and the corresponding semianalytical solution, which can be verified from the corresponding $(\zeta, \Psi)$-relationship and the cross-sectional distribution of vorticity. For higher-order multipoles the experimental data and the theoretical solutions reasonably match for intermediate values of the vorticity, but both the dented structure in the core and the extremal values in the satellites computed by Kizner et $a l .{ }^{30}$ are not reproduced by the experimental data. Recall that the cores of the observed pentapolar and higherorder multipolar vortex structures are susceptible to strong inertial oscillations, which was convincingly illustrated in Fig. 10. For that reason, we have searched for other experimental vorticity distributions that do match the dented vorticity distribution obtained by Kizner et al. ${ }^{30}$ However, such distributions were not found in the limited time interval in which the experimental multipolar vortices exist. We do not exclude the possibility of alternative semianalytical solutions similar to the anomalous structures observed in the laboratory. In pentapoles and higher-order multipoles the corotating streamlines and vorticity contours tend to become circles when approaching the vortex center. Thus both the corotating streamfunction $\Psi$ and vorticity $\zeta$ tend to become functions of the radius $r$ only, and we might conjecture that any pair of functions $\Psi$ and $\zeta$ near the vortex center would satisfy the Euler equations.

\section{SUMMARY AND CONCLUSIONS}

The time evolution of unstable isolated vortices was investigated in the laboratory, and a comparison was made between the experimentally obtained multipolar states and the semianalytical solutions for multipolar vortices derived by Kizner $e t a l .{ }^{30}$ The laboratory vortices were generated by a novel experimental technique which was inspired by the normal-mode analysis of Flierl. ${ }^{2}$ The initial vorticity distribution of the laboratory vortex was characterized by a core of single-signed vorticity and a ring of opposite-signed vorticity. Despite the deviations from a perfect piecewiseuniform vorticity distribution, the observed linear (this is, initial) evolution of the unstable monopolar vortices was close to that expected from the normal-mode analysis. Depending on the width of the annular region with respect to the diameter of the core, the nonlinear evolution of the monopolar vortices was characterized by the formation of tripolar, quadrupolar, and pentapolar vortices. To the best of the knowledge of the authors, this is the first laboratory observation of a pentapolar vortex that arises spontaneously from an initially isolated circular vortex. Also, evidence was found of the onset of hexapolar and heptapolar vortices. The experimental multipolar vortices were found to be unstable and generally broke up into multipolar vortices of lesser complexity; the latter observation being consistent with the results by Morel and Carton. ${ }^{9}$ A hexapole or a heptapole transforms into an asymmetric pentapole, which normally transforms into a quadrupole and then into a tripole. A pentapole which possesses a high degree of symmetry splits into a pair of dipoles (likewise, an unstable tripole transforms into a pair of dipoles). Although, the core vortex in this process breaks up into a pair of smaller vortices, the satellite vortices always merge to make up bigger vorticity patches. We believe this structure-simplification cascade to be allied with the inverse energy cascade. The characteristic flow 
properties of the multipolar vortices were analyzed by measuring the vorticity versus corotating streamfunction relationships as well as the cross-sectional distributions of vorticity. The flow properties of the quadrupolar vortex were in close agreement with the semianalytical model solutions by Kizner et al. ${ }^{30}$ Higher-order multipolar vortices were observed to be susceptible to strong inertial oscillations and for that reason were found to be in lesser agreement with the theoretical solutions.

\section{ACKNOWLEDGMENTS}

We are grateful to Bert Lodewijks, Stefan Nieuwenhuijsen, and Dimitry Foures for their contributions to the laboratory experiments. We are also indebted to Dr. Leon Kamp for the help with computations and to Professor M. Ungarish for helpful discussions. The Trondheim Marine Systems Research Infrastructure and, in particular, Professor T. A. McClimans are thanked for the opportunity provided to Z.K. in September 2004 to test some methodological ideas of laboratory production of shielded monopoles. Z.K. also acknowledges the support from the Israel Science Foundation Grant No. 628/06 and the financial support from the Netherlands Organization for Scientific Research (NWO) for his working visit at TUE.

${ }^{1}$ P. R. Gent and J. C. McWilliams, "The instability of barotropic circular vortices," Geophys. Astrophys. Fluid Dyn. 35, 209 (1986).

${ }^{2}$ G. R. Flierl, "On the instability of geostrophic vortices," J. Fluid Mech. 197, 349 (1988)

${ }^{3}$ X. J. Carton, G. R. Flierl, and L. M. Polvani, "The generation of tripoles from unstable axisymmetric isolated vortex structures," Europhys. Lett. 9, 339 (1989).

${ }^{4}$ X. J. Carton and J. C. McWilliams, in Mesoscale/Synoptic Coherent Structures in Geophysical Turbulence, edited by J. C. J. Nihoul and B. M. Jamart (Elsevier, Amsterdam, The Netherlands, 1989), pp. 225-244.

${ }^{5}$ B. Legras, P. Santangelo, and R. Benzi, "High-resolution numerical experiments for forced two-dimensional turbulence," Europhys. Lett. 3, 811 (1988).

${ }^{6}$ L. Polvani and X. Carton, "The tripole: A new coherent structure of incompressible two-dimensional flow," Geophys. Astrophys. Fluid Dyn. 51, 87 (1990).

${ }^{7}$ P. Orlandi and G. J. F. van Heijst, "Numerical simulations of tripolar vortices in 2D flow," Fluid Dyn. Res. 9, 179 (1992).

${ }^{8}$ X. J. Carton and B. Legras, "The life-cycle of tripoles in two-dimensional incompressible flows," J. Fluid Mech. 267, 53 (1994).

${ }^{9}$ Y. G. Morel and X. J. Carton, "Multipolar vortices in two-dimensional incompressible flows," J. Fluid Mech. 267, 23 (1994).

${ }^{10}$ F. Rossi, J. F. Lingevitch, and A. J. Bernoff, "Quasi-steady monopole and tripole attractors for relaxing vortices," Phys. Fluids 9, 2329 (1997).
${ }^{11}$ R. C. Kloosterziel and G. F. Carnevale, "On the evolution and saturation of instabilities of two-dimensional isolated circular vortices," J. Fluid Mech. 388, 217 (1999).

${ }^{12}$ G. J. F. van Heijst and R. C. Kloosterziel, "Tripolar vortices in a rotating fluid," Nature (London) 338, 569 (1989).

${ }^{13}$ G. J. F. van Heijst, R. C. Kloosterziel, and C. W. M. Williams, "Laboratory experiments on the tripolar vortex in a rotating fluid," J. Fluid Mech. 225, 301 (1991).

${ }^{14}$ R. C. Kloosterziel and G. J. F. van Heijst, "An experimental study of unstable barotropic vortices in a rotating fluid," J. Fluid Mech. 223, 1 (1991).

${ }^{15}$ J. B. Flór, W. S. S. Govers, G. J. F. van Heijst, and R. van Sluis, "Formation of a tripolar vortex in a stratified fluid," Appl. Sci. Res. 51, 405 (1993).

${ }^{16}$ A. Okamoto, K. Hara, K. Nagaoka, S. Yoshimura, J. Varnjes, M. Kono, and J. Tanaka, "Experimental observation of a tripolar vortex in a plasma," Phys. Plasmas 10, 2211 (2003)

${ }^{17}$ R. D. Pingree and B. Le Cann, "Anticyclonic eddy X91 in the southern bay of Biscay, May 1991 to February 1992," J. Geophys. Res. 97, 14353, doi:10.1029/92JC01181 (1992).

${ }^{18}$ R. Verzicco, F. Lalli, and E. Campana, "Dynamics of baroclinic vortices in rotating stratified fluid: A numerical study," Phys. Fluids 9, 419 (1997).

${ }^{19}$ G. F. Carnevale and R. C. Kloosterziel, "Emergence and evolution of triangular vortices,” J. Fluid Mech. 259, 305 (1994).

${ }^{20}$ T. B. Mitchell and C. F. Driscoll, "Symmetrization of vortices by beatwave damping," Phys. Rev. Lett. 73, 2196 (1994).

${ }^{21}$ X.-P. Huang, K. S. Fine, and C. F. Driscoll, "Coherent vorticity holes from 2D turbulence decaying in a background shear flow," Phys. Rev. Lett. 74, 4424 (1995).

${ }^{22}$ M. Beckers and G. J. F. van Heijst, "The observation of a triangular vortex in a rotating fluid," Fluid Dyn. Res. 22, 265 (1998).

${ }^{23}$ G. K. Morikawa and E. V. Swenson, "Interacting motion of rectilinear geostrophic vortices," Phys. Fluids 14, 1058 (1971).

${ }^{24}$ B. Eckhardt, "Integrable four vortex motion," Phys. Fluids 31, 2796 (1988).

${ }^{25} \mathrm{H}$. Aref, "On the equilibium and stability of a row of point vortices," J. Fluid Mech. 290, 167 (1995).

${ }^{26} \mathrm{H}$. Aref and M. A. Stremler, "Four-vortex motion with zero total circulation and impulse," Phys. Fluids 11, 3704 (1999).

${ }^{27}$ Z. Kizner and R. Khvoles, "The tripolar vortex: Experimental evidence and explicit solutions," Phys. Rev. E 70, 016307 (2004).

${ }^{28}$ Z. Kizner and R. Khvoles, "Two variations on the theme of LambChaplygin: Supersmooth dipole and rotating multipoles," Regular Chaotic Dyn. 9, 509 (2004).

${ }^{29}$ R. C. Kloosterziel and G. J. F. van Heijst, "The evolution of stable barotropic vortices in a rotating free-surface fluid," J. Fluid Mech. 239, 607 (1992).

${ }^{30}$ Z. Kizner, R. Khvoles, and J. C. McWilliams, "Rotating multipoles on the $f$ - and $\gamma$-planes," Phys. Fluids 19, 016603 (2007).

${ }^{31}$ N. J. Zabusky, M. H. Hughes, and K. V. Roberts, "Contour dynamics for the Euler equations in two dimensions," J. Comput. Phys. 30, 96 (1979).

${ }^{32}$ K. Stewartson, "On almost rigid rotations," J. Fluid Mech. 3, 17 (1957).

${ }^{33}$ M. Duran-Matute, L. P. J. Kamp, R. R. Trieling, and G. J. F. van Heijst, "Inertial oscillations in a confined monopolar vortex subject to background rotation," Phys. Fluids 21, 116602 (2009). 